\title{
BMJ Open Healing Right Way: study protocol for a stepped wedge cluster randomised controlled trial to enhance rehabilitation services and improve quality of life in Aboriginal Australians after brain injury
}

\author{
Elizabeth Armstrong (D) , ${ }^{1}$ Juli Coffin, ${ }^{2}$ Deborah Hersh, ${ }^{1}$ \\ Judith M Katzenellenbogen (D) , ${ }^{3}$ Sandra Thompson, ${ }^{4}$ Leon Flicker, ${ }^{5}$ \\ Meaghan McAllister, ${ }^{1}$ Dominique A Cadilhac (D) , ${ }^{6}$ Tapan Rai (D) , \\ Erin Godecke (D) , ${ }^{1}$ Colleen Hayward, ${ }^{8}$ Graeme J Hankey, ${ }^{9}$ Neil Drew, ${ }^{8}$ Ivan Lin, ${ }^{10,11}$ \\ Deborah Woods, ${ }^{11}$ Natalie Ciccone ${ }^{1}$
}

To cite: Armstrong E, Coffin J, Hersh D, et al. Healing Right Way: study protocol for a stepped wedge cluster randomised controlled trial to enhance rehabilitation services and improve quality of life in Aboriginal Australians after brain injury. BMJ Open 2021;11:e045898. doi:10.1136/ bmjopen-2020-045898

- Prepublication history for this paper is available online. To view these files, please visit the journal online (http://dx.doi. org/10.1136/bmjopen-2020045898).

Received 17 October 2020 Accepted 31 August 2021

\section{Linked}

- http://dx.doi.org/10.1136/ bmjopen-2020-046042

Check for updates

(c) Author(s) (or their employer(s)) 2021. Re-use permitted under CC BY-NC. No commercial re-use. See rights and permissions. Published by BMJ.

For numbered affiliations see end of article.

Correspondence to Professor Elizabeth Armstrong; b.armstrong@ecu.edu.au

\section{ABSTRACT}

Introduction Despite higher incidence of brain injury among Aboriginal compared with non-Aboriginal

Australians, suboptimal engagement exists between rehabilitation services and Aboriginal brain injury survivors. Aboriginal patients often feel culturally insecure in hospital and navigation of services post discharge is complex. Health professionals report feeling ill-equipped working with Aboriginal patients. This study will test the impact of a research-informed culturally secure intervention model for Aboriginal people with brain injury.

Methods and analysis Design: Stepped wedge cluster randomised control trial design; intervention sequentially introduced at four pairs of healthcare sites across Western Australia at 26-week intervals.

Recruitment: Aboriginal participants aged $\geq 18$ years within 4 weeks of an acute stroke or traumatic brain injury. Intervention: (1) Cultural security training for hospital staff and (2) local, trial-specific, Aboriginal Brain Injury Coordinators supporting participants.

Primary outcome: Quality-of-life using EuroQ0L-5D-3L (European Quality of Life scale, five dimensions, three severity levels) Visual Analogue Scale score at 26 weeks post injury. Recruitment of 312 participants is estimated to detect a difference of 15 points with $80 \%$ power at the $5 \%$ significance level. A linear mixed model will be used to assess the between-condition difference.

Secondary outcome measures: Modified Rankin Scale, Functional Independence Measure, Modified Caregiver Strain Index, Hospital Anxiety and Depression Scale at 12 and 26 weeks post injury, rehabilitation occasions of service received, hospital compliance with minimum care processes by 26 weeks post injury, acceptability of Intervention Package, feasibility of Aboriginal Brain Injury Coordinator role.

Evaluations: An economic evaluation will determine the potential cost-effectiveness of the intervention. Process evaluation will document fidelity to study processes and capture changing contexts including barriers to intervention implementation and acceptability/feasibility of
Strengths and limitations of this study

- This is the first stepped-wedge randomised control trial to explore the effects of an intervention on access to rehabilitation services and outcomes for Aboriginal survivors of brain injury.

- The study involves strong collaboration between Aboriginal and non-Aboriginal researchers and health service providers to maximise the opportunity for translation into policy and practice.

- We will gather the first comprehensive data on resource utilisation, costs and outcomes for an economic evaluation of care facilitation roles applicable to the study cohort.

- Ongoing service developments external to the study may influence the outcomes of participants; while these contextual factors will be monitored and reported as part of the study's process evaluation, their effects are beyond the control of the study.

- By the nature of the stepped-wedge design, with hospital and region-wide interventions difficult to conceal, blinding to intervention may not always be possible.

the intervention through participant questionnaires at 12 and 26 weeks.

Ethics and dissemination The study has approvals from Aboriginal, university and health services human research ethics committees. Findings will be disseminated through stakeholder reports, participant workshops, peer-reviewed journal articles and conference papers.

Trial registration number ACTRN12618000139279.

\section{INTRODUCTION}

Background and rationale

The Aboriginal and Torres Strait Islander population (hereafter respectfully referred to as Aboriginal), Australia's First Nations 
peoples, constitutes $3.3 \%$ of the Australian population ${ }^{1}$ and consists of many different cultural groups, with more than 120 separate Aboriginal languages spoken (240 at the time of colonisation). ${ }^{2}$ Aboriginal Australians are overall younger than other Australians (median age 23 vs 38 years), ${ }^{1}$, with health inequities well-documented. ${ }^{3}$ The incidence of brain injury is significantly greater in Aboriginal than non-Aboriginal Australians, with stroke and traumatic brain injury (TBI) occurring up to three times more frequently ${ }^{4-8}$ and functional dependence at hospital discharge three times more likely. ${ }^{4}$ Motor, communication, sensory and cognitive deficits all adversely affect long-term quality of life, including employment status and prospects, family relationships, social participation and mental health. ${ }^{9-12}$ Ongoing engagement between Aboriginal brain injury survivors and mainstream hospital-based rehabilitation services is limited, with complex service pathways to navigate post discharge. ${ }^{1314}$ This results in confusion, communication breakdown and lack of support for brain injury survivors, their families and communities. Poor service leads to ongoing challenges and re-hospitalisations that incur additional health system costs. ${ }^{1516}$

Despite some recent improvements in access to acute services such as stroke units and thrombolysis ${ }^{17}$ multiple issues persist among those requiring subsequent care: lack of practical, understandable information regarding brain injury; limited availability of Aboriginal Liaison Officers to assist with navigating services; lack of interpreter services; racist attitudes/assumptions of some healthcare providers; and underservicing of rural and remote areas. ${ }^{118-21}$ Many health professionals feel underprepared to work with Aboriginal patients in a culturally secure manner ${ }^{21-23}$ and few people consult Aboriginal Health Services specifically for brain injury-related concerns. ${ }^{18}$

The current trial will test the impact of a researchinformed culturally secure ${ }^{21}$ intervention model for Aboriginal people with brain injury in Western Australia

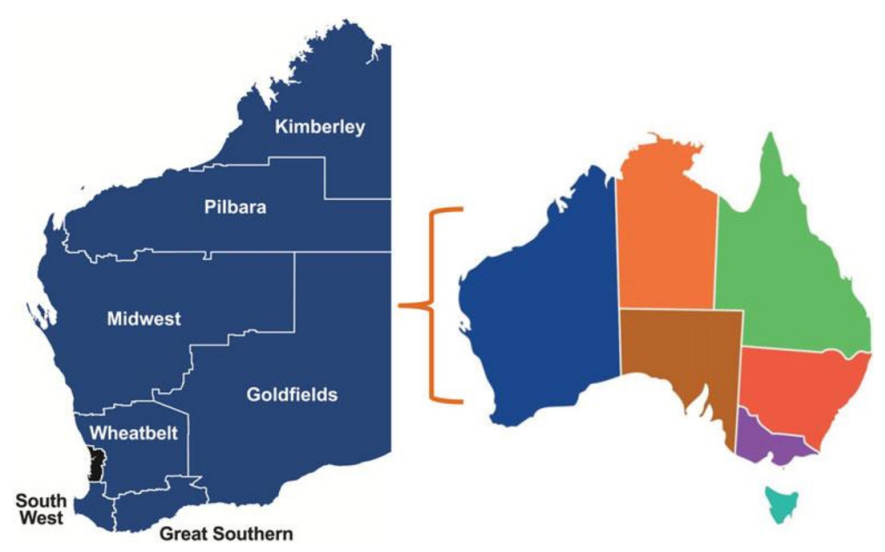

Figure 1 (Colour online) A map of the state of Western Australia, with major regions highlighted, in relation to the whole of Australia. (Image of state regions reproduced with the permission of the Western Australian Country Health Service.)
(WA) (see figure 1). Cultural security refers to ensuring that Aboriginal cultural values, world views and ways of working are incorporated at each stage of care for an Aboriginal person and that services will not compromise the legitimate cultural rights, values and expectations of Aboriginal people. ${ }^{21}$ The trial addresses the systemic challenges identified in our research, using existing resources where possible and developing a site and service-specific sustainable evidence-based approach. Due to the chronic consequences of brain injury, the proposed intervention package follows a Chronic Care Model (CCM) ${ }^{24}$ demonstrated to be successful in the management of other chronic conditions. The CCM has been modified for the purpose of this trial to incorporate specific components related to brain injury. The study will occur in partnership with state government-funded health services and Aboriginal Community Controlled Health Services, as well as national policymakers.

A stepped-wedge cluster design was chosen given: (1) the population under focus is under-represented in rehabilitation services, is vulnerable in terms of comorbidities and has poorer overall health outcomes. Withholding an additional service intervention likely to do more good than harm at particular sites within a trial would pose an ethical dilemma; (2) the design still includes a control condition, making comparisons of intervention effectiveness possible; (3) the sequential introduction of the intervention to clusters facilitates the assessment of changes in service standards through secular trends over time; (4) a concurrent roll-out of the intervention across a large geographical area (975 685 square miles), encompassing rural and remote areas, was not feasible.

\section{Aboriginal research framework}

The trial will employ principles aligned with an Aboriginal Research Framework as recommended for health contexts ${ }^{25}$ and incorporates central notions from Indigenous Standpoint Theory ${ }^{2627}$ which relate to research with Indigenous peoples, namely: inclusion and leadership by Aboriginal researchers; acceptance of colonisation as a social determinant of health/disability; acknowledgement of the diversity of Aboriginal communities; use of local languages; and Aboriginal community capacity building.

\section{Objectives}

The aims of this trial are to: (1) improve delivery of rehabilitation services to Aboriginal people post brain injury (stroke and traumatic brain injury), (2) improve overall health outcomes of this group, (3) conduct an economic evaluation to support the business case for resourcing future rehabilitation services if the intervention is determined to be cost-effective and (4) explore the acceptability of the intervention from the perspectives of health professionals and Aboriginal participants involved, and to use this information to assist in interpretation and translation of findings. 
Our primary hypothesis is that compared with usual care (UC), implementation of the proposed intervention package (IP) will result in at least a 15-point higher score on the EuroQOL-5D-3L ${ }^{28}$ (European Quality of Life scale, comprised of five dimensions: Mobility, Self-care, Usual activity, Pain and discomfort, Anxiety and depression; and 3 severity levels) Visual Analogue Scale (VAS) at 26 weeks post injury. Our secondary hypotheses are:

1. Compared with UC, implementation of the IP will result in improvement in service delivery at 12 and 26 weeks post injury as measured by increased occasions of service.

2. Compared with UC, implementation of the IP will result in improvement in service delivery at 12 and 26 weeks post injury as measured by indicators of essential processes of care.

3. Compared with UC, implementation of the IP will result in reduction in disability (modified Rankin Scale $(\mathrm{mRS}))^{29}$ and greater independence (Functional Independence Measure (FIM) $)^{30}$ at 12 and 26 weeks post injury.

4. Compared with UC, implementation of the IP will result in less carer burden (Modified Caregiver Strain Index ${ }^{31}$ and less brain injury survivor anxiety and depression (Hospital Anxiety and Depression Scale) ${ }^{32}$ at 12 and 26 weeks post injury.

5. The culturally sensitive IP will be cost-effective (benefits gained will justify costs for delivering the intervention; or lead to cost-offsets from less severe disease) when compared with UC 26 weeks post injury.

6 . The IP will be acceptable to health professionals and Aboriginal participants and their families, and the Aboriginal Brain Injury Coordinator role is feasible.

\section{METHODS}

The reporting of the methods aligns with the Standard Protocol Items: Recommendations for Interventional Trials guidelines ${ }^{33}$ for clinical trial protocols and incorporates relevant details from the Consolidated Standards of Reporting Trials extension statement on the reporting of stepped-wedge cluster randomised control trials (CRCTs) ${ }^{34}$

\section{Trial design}

As noted above, a stepped-wedge CRCT design ${ }^{35}$ will be used, with paired healthcare sites functioning as clusters (see figure 2). The pairing was done to prevent contamination between sites since it is common for patients to be transferred between the rural and metropolitan sites that are paired together. The pairing also enables adjustment for differences in numbers of participants recruited in the rural/metropolitan site pairs. Twenty-six weeks of baseline control data will be obtained prior to implementation of the intervention, which will be introduced sequentially to all sites at 26 -week intervals. Control data will continue to be collected at each site until the intervention commences. The intervention

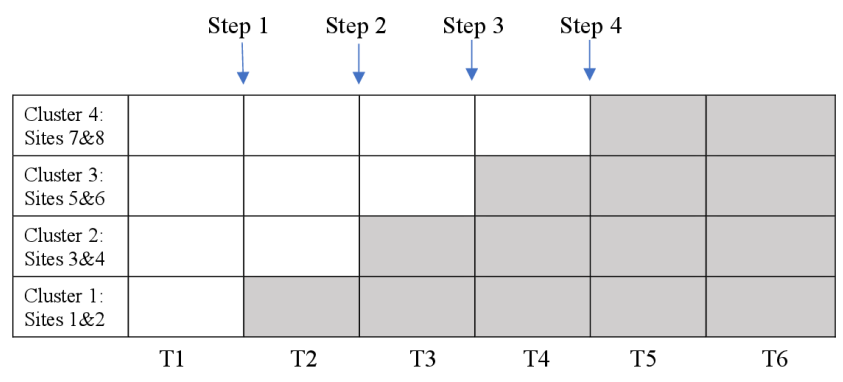

Control condition

Intervention condition

$\mathrm{T} 1=$ Time period 1 ( 6 months duration $)$ etc

Figure 2 Stepped-wedge design.

will continue until all sites have received the intervention for at least 52 weeks. The sequence of receipt of intervention will be determined by randomisation of clusters.

This research is a complex intervention due to the multiple components involved, the varied sites that incorporate diverging local contexts, potential contamination across sites due to movement of some participants between rural and metropolitan hospitals within the study period and potential clinical service changes during the intervention period. A process evaluation ${ }^{36}$ will be undertaken alongside the measurement and analysis of the intervention and primary outcomes as per the Template for Intervention, Description and Replication (TIDieR) checklist. $^{37}$

A Steering Committee will oversee the study, consisting of the chief investigators (CIs), assisted by the trial manager. The Data Collection and Management Team consists of six CIs, assisted by the trial data and operations manager. Working parties for both intervention conditions will consist of CIs and associate investigators experienced in cultural security training and Aboriginal workforce organisation. A Design Working Party, consisting of the principal investigator and six other CIs, will oversee any protocol amendments.

\section{Participants}

Aboriginal people aged $\geq 18$ years who have suffered an acute stroke or TBI will be recruited within 4 weeks post injury. Specific inclusion and exclusion criteria are presented in figure 3 along with relevant definitions. ${ }^{38-41}$

Participating sites will include eight acute hospital sites across WA (four metropolitan and four regional). The sites were chosen as the metropolitan hospitals constitute the three major tertiary hospitals in WA and a secondary hospital with an acute stroke unit. The regional hospitals constitute four large regional centres. All sites were known to have significant numbers of Aboriginal people admitted. Transfer across hospitals within WA is anticipated, and 'step-down', that is, non-acute rehabilitation sites will be involved (ethics approval obtained) for participant follow-up but not recruitment. 


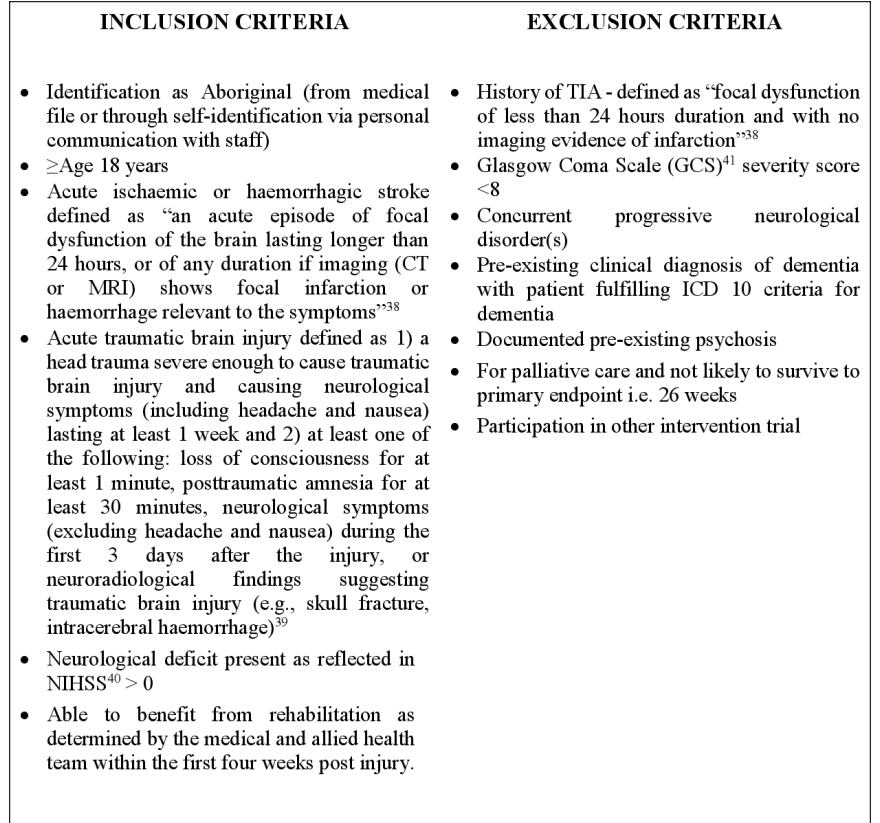

Figure 3 Inclusion and exclusion criteria. TIA, transient ischaemic attack; ICD-10, International Classification of Diseases, Tenth Revision; NIHSS, National Institutes of Health Stroke Scale.

\section{Interventions}

The intervention periods will vary across sites (between 1 and 2.5 years) depending on cluster randomisation under the stepped-wedge design. The intervention will consist of two components-(1) cultural security training (CST) for hospital staff, and (2) introduction of an Aboriginal Brain Injury Coordinator (ABIC) at each site employed for 1 day/week.

\section{Cultural security training of hospital staff}

Twenty health professionals at each site (nursing, medical and allied health) will complete the initial CST of 3 hours face-to-face followed by 3 hours online, focusing on challenges surrounding brain injury. The face-to-face sessions will be co-facilitated by a local Aboriginal cultural security trainer and a member of the research team. The online component must be completed within 3-4 weeks of completion of the face-to-face component. The training will be offered at each site every 6 months to address staff attrition. The materials and format of the training are based on extensive consultation with Aboriginal people after brain injury and their families, ${ }^{11} 12202142$ Aboriginal and non-Aboriginal health professionals and Aboriginal experts in cultural security, including the second author. They are based on principles of cultural security, ${ }^{21}$ an Aboriginal model of health ${ }^{43}$ and 'clinical yarning'. ${ }^{42}$ Videos of Aboriginal people who have experienced brain injury discussing their hospital experiences are central, with reflective exercises and case studies embedded.

\section{Aboriginal Brain Injury Coordinator}

An Aboriginal person with a relevant health or community care background will be employed 1 day/week at each of the trial sites as an ABIC. The role is based on the Neurological Nurse and Neurocare model of the Neurological Council of Western Australia (NCWA) ${ }^{44}-\mathrm{a}$ community neurological nursing service. The ABIC will see participants in hospital and up until 26 weeks post injury onset, providing education, support, liaison and advocacy services to participants and their families. The ABIC will receive 12 hours of training and ongoing support from the partner clinical team (NCWA) and the research team. ABICs will be located in the hospital, the local Aboriginal Community Controlled Health Service, or offices of NCWA, depending on site preference. Follow-up of participants will occur through face-to-face, phone or telehealth contact as agreed.

An intervention protocol provided only to the ABICs outlines prescribed activities that must be performed within specific time frames.

\section{Intervention integrity}

Adherence to content and staff attendance at the faceto-face component of the CST will be recorded by the researcher involved in the site-training. Completion of the online training component will be recorded on the secure website containing the online materials. REDCap (Research Electronic Data Capture) data entry related to the ABIC role will be monitored on a monthly basis. Feedback will be provided to the ABIC if data is missing or prescribed activities outlined in the intervention protocol have not been performed.

\section{Outcomes}

\section{Primary outcome}

The primary outcome measure is the EuroQOL-5D-3L VAS score administered at 26 weeks post injury. The EQ-5D ${ }^{28}$ has previously been used with stroke and TBI survivors, ${ }^{45} 46$ validated in different countries and cultural settings, and can be administered by face-to-face or telephone interview, or as a self or proxy mail-out. The VAS involves a 100-point vertical scale measuring quality of life on the day of administration as perceived by participants. The scale endpoints are labelled 'Best imaginable health state' and 'Worst imaginable health state.'

\section{Secondary outcome measures}

These relate to the health outcomes of post-stroke symptoms and functional status (mRS), ${ }^{29}$ functional independence (FIM), ${ }^{30}$ burden of care (Modified Caregiver Strain Index), ${ }^{31}$ anxiety and depression (Hospital Anxiety and Depression Scale) ${ }^{32}$ and clinical service provision (allied health rehabilitation sessions and minimum process of care indicators). Figure 4 outlines the minimum processes of care indicators developed for this study, based on clinical guidelines ${ }^{47}$ and best practice statements. ${ }^{48}$ Participant (Aboriginal people post stroke/TBI) satisfaction, reflecting acceptability of hospital services and the ABIC services will be assessed through questionnaires administered either face-to-face or by phone/telehealth facility and incorporated into the process evaluation. Staff 
- Patient assessed by allied health within 48 hours of admission

- Aboriginal Liaison Officer involved during inpatient stay

- Language(s) spoken noted in medical file

- Interpreter used if Aboriginal language is participant's primary language

- Telephone or face to face contact with family made by any allied health staff during inpatient stay

- Aboriginal brain injury educational resource provided during inpatient stay

- Inpatient allied health service provided (any discipline)

- Discharge plan developed with patient and family at family conference

- Outpatient allied health service provided (any discipline)

- In the case of transfer from metropolitan to rural service, verbal contact made by the metropolitan hospital with local rural service provider (hospital and local Aboriginal Medical Service)

- In the case of transfer from metropolitan to rural service, discharge report from metropolitan hospital sent to rural service provider (hospital and local Aboriginal Medical Service)

Figure 4 Minimum processes of care.

satisfaction with the CST will be assessed through questionnaires administered both face-to-face and online. Feasibility of the ABIC role will be assessed through the process evaluation service data collection.

Resource utilisation and costs: see data collection section below.

\section{Data collection}

Patient outcomes

Baseline assessments will be undertaken by qualified assessors with an Aboriginal Liaison Officer present where possible. Demographic information (age, gender, language group, place of residence) will be collected at baseline assessment. Follow-up assessments will be undertaken by an independent qualified blinded assessor at weeks 12 and 26 post injury. All assessments will take place at participants' place of residence, in the hospital or Aboriginal Community Controlled Health Service clinic as convenient for the participant, or by phone/telehealth as appropriate. Any deviation from the prescribed protocols will be recorded as a protocol deviation.

\section{Service data}

Occasions of allied health rehabilitation service across the 26 weeks post injury will be collected through a medical file review process and through access to hospital administrative data systems. Minimum process of care indicators will capture data on whether identified key processes occurred across the first 26 weeks post injury. The data collection will be undertaken by an independent blinded auditor.

The activities of the ABIC involving direct and indirect contact with participants will be recorded in the electronic case report form.

\section{Process evaluation data}

Data will be collected to enable evaluation of potential causal and overall contextual factors related to the outcomes of the study, as recommended by Medical Research Council (MRC) ${ }^{49}$ and as included in the TIDieR checklist. ${ }^{37}$ Ongoing site-specific descriptive information will be collected related to the hospital context, for example, general staffing levels, staff turnover, policy changes. Factors related to the impact of the CST including staff satisfaction with, and perceived

\begin{tabular}{|c|c|c|c|}
\hline Assessment & \begin{tabular}{l}
\multicolumn{1}{c}{ Baseline } \\
Between Day 2 \\
and Day 28 post \\
injury
\end{tabular} & $\begin{array}{l}\text { Week } 12 \\
12 \text { weeks post } \\
\text { injury }+/-7 \text { days }\end{array}$ & $\begin{array}{l}\text { Week } 26 \\
26 \text { weeks post } \\
\text { injury }+/-14 \\
\text { days }\end{array}$ \\
\hline Screening/Eligibility & $\mathrm{X}$ & & \\
\hline Consent & $\mathbf{X}$ & & \\
\hline Demographics & $\mathbf{X}$ & & \\
\hline Past medical History & $\mathbf{X}$ & & \\
\hline Brain injury subtypes & $\mathbf{X}$ & & \\
\hline NIHSS & $\mathbf{X}$ & & \\
\hline GCS & $\mathbf{X}$ & & \\
\hline mRS & $\mathbf{X}$ & $\mathbf{X}$ & $\mathbf{X}$ \\
\hline FIM $^{\mathrm{TM}}$ & $\mathrm{X}$ & $\mathbf{X}$ & $\mathrm{X}$ \\
\hline $\begin{array}{l}\text { Modified Caregiver } \\
\text { Strain index }\end{array}$ & & $\mathbf{X}$ & $\mathbf{X}$ \\
\hline $\begin{array}{l}\text { Hospital Anxiety and } \\
\text { Depression Scale }\end{array}$ & $\mathbf{X}$ & $\mathbf{X}$ & $\mathbf{X}$ \\
\hline EuroQol-5D-3L & & $\mathbf{X}$ & $\mathbf{X}$ \\
\hline $\begin{array}{l}\text { Allied health Occasions } \\
\text { of Service }\end{array}$ & & $\mathbf{X}$ & $\mathbf{X}$ \\
\hline $\begin{array}{l}\text { Minimum processes of } \\
\text { care }\end{array}$ & & $\mathbf{X}$ & $\mathbf{X}$ \\
\hline Resource Utilisation & & $\mathbf{X}$ & $\mathbf{X}$ \\
\hline Adverse Events & & $\mathbf{X}$ & $\mathrm{X}$ \\
\hline SAEs & & $\mathbf{X}$ & $\mathbf{X}$ \\
\hline
\end{tabular}

Figure 5 Schedule of assessments. NIHSS, National Institutes of Health Stroke Scale; GCS, Glasgow Coma Scale; FIM, Functional Independence Measure; mRS, modified

Rankin Scale; EuroQol-5D-3L, European Quality of Life scale, comprised of five dimensions and three severity levels; SAEs, serious adverse events.

usefulness of training will be measured through evaluation questionnaires. A questionnaire on participants' hospital experiences and brain injury-related services received will be administered by blinded assessors at 12 and 26 weeks. A questionnaire addressing participation satisfaction with the ABIC services will also be administered. A detailed outline of the process evaluation will be published separately. ${ }^{36}$

\section{Resource utilisation}

Resource utilisation information will be collected using programme administrative data, hospital data captured from medical records and self-report using a standardised questionnaire. General service data will be collected for all patients at the week 12 and 26 visits regarding inpatient and outpatient rehabilitation-related sessions (rehabilitation specialist, allied health sessions). Unit prices for participant self-reported resource use items will be obtained from published sources or be derived directly from the trial in terms of programme delivery costs and hospital care.

A summary of the data collection schedule is provided in figure 5 .

\section{Sample size}

To estimate the required sample size we calculated an effect size of $\mathrm{d}=0.6$, based on an anticipated improvement of 15 points on the EuroQOL-5D VAS, with an SD of $25 .{ }^{46}$ GPower V.3.1 ${ }^{50}$ estimated that a total of 90 participants required to detect this difference with $80 \%$ power and $\alpha=0.05$. After adjusting for the design effect ${ }^{51}$ for a four-step stepped-wedge design with intra-class correlation co-efficient $(\mathrm{ICC})=0.08$, one baseline step and one follow-up step, we estimated the total sample size required as 312 . 


\section{Participant completion and discontinuation}

Participants have completed the study when the final follow-up visit is complete (week 26) and all data pertaining to this visit have been submitted to the study sponsor. Participants' data will be withdrawn from the study if they withdraw consent, or if it is determined that trial involvement poses a health or safety risk to the participant. Data collected up until the time of withdrawal will be used. Withdrawn participants will not be replaced. Retention of participants will be maximised by a letter/email/telephone call in advance of the follow-up assessment date to remind participants of their enrolment in the study and facilitate organisation of follow-up assessments.

\section{RANDOMISATION}

\section{Sequence generation}

Clusters will be randomised at the beginning of the trial to determine the sequencing of the introduction of the intervention. Numbers of participants recruited in metropolitan sites are anticipated to be higher than in regional sites due to potential transfer of patients to tertiary metropolitan hospitals for early treatment post stroke/TBI. To minimise potential differences in participant numbers across steps, one metropolitan and one regional site will be paired prior to randomisation, then these pairs of sites will be randomised. The pairings were primarily determined by existing stroke pathways between rural and metropolitan regions. Intervention commencement time will be assigned through use of a computer-generated sequence of random numbers. The process will allocate sites to one of four commencement periods (figure 2).

\section{Implementation}

The trial statistician will undertake the randomisation process and will be unaware of the identity/location of the sites involved.

All Aboriginal stroke and patients with TBI $\geq 18$ years of age admitted to hospital will be screened for study inclusion as per eligibility criteria. Those meeting criteria will be approached to participate by the hospital investigator, preferably with the assistance of a hospital-based Aboriginal Liaison Officer. If the person agrees to provision of their name to the trial team, a team member will discuss the trial further with the person and obtain informed consent, involving an interpreter as needed. 'Aphasia friendly' information will be used to accommodate patients with communication disorders, cognitive issues and/or limited literacy. For patients with very severe impairment, we will seek assent from the patient as well as proxy consent for research involvement from a person responsible (family member, friend, long-term carer or guardian) for the participant. Consent to participate in the CST will be obtained from all hospital staff completing the training. The same process will occur regardless of control/intervention phase of the trial.

\section{Blinding}

All assessors will be independent of the researchers involved in the intervention or trial. However, assessors in rural areas may be aware of whether their local hospital is in intervention or control phase. Therefore, it is not possible to blind all the assessors, patients or most investigators with respect to whether the patients received intervention or not. Follow-up assessors will be blinded to the baseline assessment of any given participant.

\section{Data management}

\section{Electronic case report form}

An electronic case report form (eCRF) will be completed for each participant summarising all clinical screening and study data. Participants will only be recorded by their participant number and initials in order to retain participant confidentiality. Data will be collected and managed using REDCap electronic data capturing tool. The trial manager and trial data and operations manager will monitor site compliance with study procedures and completion of the eCRFs. Site visits conducted independently of the investigator team will include review of medical records, comparison with source documents and observation and discussion of the conduct of the study with the site contact.

\section{Statistical methods}

Unadjusted analyses

Descriptive statistics (consisting of the mean and SD or median and IQR or frequency and per cent) will be reported for all available data on the outcome measures at baseline, 12 weeks and 26 weeks. Precise details on the tables and descriptive statistics to be presented will be published separately in a statistical analysis plan (SAP), which will be finalised before the database is locked.

\section{Adjusted analyses}

The primary analysis will be on an intention-to-treat basis with each participant allocated to the site/treatment that he/she was originally recruited. If the amount of missing data at the primary endpoint exceeds $10 \%$, multiple imputation will be performed under the assumption that data is missing at random. A sensitivity analysis considering assumptions about data missing not at random will be conducted. Additional details on the missing data imputation will be provided in the SAP.

\section{Primary outcome analysis}

A mixed effects linear regression model will assess the between-condition difference on EuroQOL-5D-3L VAS score at 26 weeks post injury.

\section{Secondary outcomes analyses}

A mixed-effect regression model will be used to assess between-condition differences for each of the outcomes: occasions of service (1), functional independence (3), Modified Carer Strain Index and Hospital Anxiety and Depression Scale (4), at 12 and 26 weeks post injury. Minimum process of care indicators (2) will be 
dichotomised (achieved/not achieved). Similarly, mRS will be dichotomised as good outcome (mRS 0-2) and poor outcome (mRS 3-6). A mixed-effect logistic regression model will assess between-condition differences on each of these binary variables at 12- and 26 weeks postinjury. Additional details about these models will be provided in the SAP; these will include plans for alternative models in case any of the proposed models fail to converge. Descriptive statistics for participant satisfaction, reflecting acceptability of hospital services, staff satisfaction with the CST and feasibility of the ABIC role will be presented in terms of frequencies and percentages, but these outcomes will not be subject to any statistical modelling. A more in-depth analysis of these variables will be conducted qualitatively as part of our process evaluation, which is outlined in a separate paper.

\section{Interim analysis}

No interim analysis of efficacy is planned for the trial. However, if the Data Safety Monitoring Committee (DSMC-see below) members develop a safety concern following review of trial data (eg, an imbalance of serious adverse events (SAEs) between conditions), the DSMC may request an interim safety or efficacy analysis of the data.

Only the trial manager, trial data manager, trial statistician and an independent statistician will have access to the final locked database. Investigators will view the results of the final analyses, but will not have access to raw data.

\section{Economic analysis}

Cost description analyses of each comparator condition will be detailed using a decision-analytical model. Intervention delivery costs will be included for the intervention condition. The incremental (net) costs and benefits of the intervention (ie, quality-adjusted life years (QALYs) gained derived from EQ-5D results) compared with control will be determined. Sensitivity and probabilistic multivariable uncertainty analyses will be performed to assess the robustness of results. The intervention will be judged cost-effective if the incremental cost per QALY gained is $<$ AUD $\$ 50000$ (ie, the willingness-to-pay threshold). Cost-effectiveness acceptability curves will also be generated as a function of describing potential willingness-to-pay. This method provides a measure of magnitude and uncertainty of cost-effectiveness, expressed as a probability statement meaningful to policymakers. The statistical analysis plan and reporting of the economic evaluation will be guided by the Consolidated Health Economic Evaluation Reporting Standards statement ${ }^{52}$ and the recommendations from the European Stroke Organisation Health Economic Working Group. ${ }^{53}$

\section{Harm}

An independent DSMC will regularly review the study data and make recommendations to the trial team. The Committee will consist of three members (at least one Aboriginal member) who are independent of the trial, have different disciplinary backgrounds and who have experience in the management of patients and the conduct of clinical trials. Ongoing review of trial safety data will be held at agreed intervals as determined by the DSMC members. Discussion about the final data will occur with the trial team at trial completion. The Steering Committee will make the final decision on whether to stop the trial due to safety concerns or ascertainment of undue risks to participants.

\section{Safety assessments}

Adverse events (AEs) that are possibly, probably or definitely attributable to the intervention will be reported via the eCRF. The investigator and designated study personnel will monitor each participant for AEs during the study. AEs that meet the criteria for serious, are considered SAEs and will be reported throughout the trial.

\section{Patient and public involvement}

Healing Right Way is informed by previous studies ${ }^{11} 18-2054$ that have prioritised the voices of Aboriginal people with brain injury and their families, as well as those of Aboriginal and non-Aboriginal health service providers. Healing Right Way involves collaboration between a team of Aboriginal and non-Aboriginal researchers and the range of service providers indicated above. An Aboriginal Reference Group will guide and oversee the research to ensure the study is conducted according to principles of cultural security. ${ }^{21}$ It will provide advice regarding participant recruitment, relevant human and practical resources (eg, interpreters), community feedback from the trial and general cultural and ethical issues. The Reference Group will meet twice per year and provide other input as needed.

\section{ETHICS AND DISSEMINATION}

The protocol was approved by Royal Perth Hospital (the central ethics committee for the study \#0000000125), St John of God Hospital (\#1198), Edith Cowan University (\#17291) and the WA Aboriginal Health (\#794) Human Research Ethics Committees. The principal investigator, or her delegate, will be responsible for reporting any SAEs to the Ethics Committees as soon as possible, and in accordance with the guidelines of the Ethics Committee. Any protocol modifications will be approved by all ethics committees and conveyed to all partner investigators at each site.

This study will be carried out according to the Declaration of Helsinki, the NHMRC National Statement on Ethical Conduct in Research Involving Humans (2007; updated 2018) ${ }^{55}$ and the Notes for Guidance on Good Clinical Practice (GCP) as adopted by the Australian Therapeutic Goods Administration (2000) (CPMP/ ICH/135/95), the ICH GCP guidelines. ${ }^{56}$ Conducting research with and within Aboriginal communities carries significant responsibilities central to the research 
practices of the study. An Aboriginal research framework ${ }^{25}$ incorporating principles of cultural security ${ }^{21}$ will be used in this study, which will align with the National Health \& Medical Research Council 'Ethical conduct for research with Aboriginal and Torres Strait Islander Peoples and communities: Guidelines for researchers and stakeholders 2018, ${ }^{57}$ 'Keeping Research on Track II $2018^{, 58}$ and the Australian Institute for Aboriginal and Torres Strait Islander Studies' Guidelines for Ethical Research in Australian Indigenous Studies. ${ }^{59}$ All data will be de-identified and remain confidential throughout and subsequent to the trial completion.

Knowledge sharing and translation will be guided by a Knowledge Translation and Exchange Plan to guide key messaging and to identify audiences and enablers. Transfer of knowledge is planned through feedback sessions to all participants, to be given in multiple formats including workshops, community meetings, written reports and social media. More formal transfer will occur through input into national clinical guidelines, ongoing liaison with policymakers and dissemination of results through publications and conferences. Authorship of publications will include all research team investigators and project partners, with all publications approved by the Steering Committee.

\section{DISCUSSION}

This landmark study provides a novel, integrated complex intervention across a large geographical area in an underserviced population in real-life settings. The trial will provide vital information to shape much-needed service improvements for Aboriginal people following brain injury including economic information to support the planning and sustainability of future services.

\section{Author affiliations}

${ }^{1}$ School of Medical and Health Sciences, Edith Cowan University, Joondalup, Western Australia, Australia

${ }^{2}$ Telethon Kids Institute, Broome, Western Australia, Australia

${ }^{3}$ School of Population and Global Health, University of Western Australia, Crawley, Western Australia, Australia

${ }^{4}$ Western Australian Centre for Rural Health, The University of Western Australia, Perth, Western Australia, Australia

${ }^{5}$ WA Centre for Health and Ageing, University of Western Australia, Perth, Western Australia, Australia

${ }^{6}$ Department of Medicine, Monash University, Clayton, Victoria, Australia

${ }^{7}$ School of Mathematical and Physical Sciences, University of Technology Sydney,

Sydney, New South Wales, Australia

${ }^{8}$ School of Kurongkurl Katitjin, Edith Cowan University, Mount Lawley, Western Australia, Australia

${ }^{9}$ School of Medicine and Pharmacology, The University of Western Australia, Perth, Western Australia, Australia

${ }^{10}$ Western Australian Centre for Rural Health, University of Western Australia Geraldton Campus, Geraldton, Western Australia, Australia

${ }^{11}$ Geraldton Regional Aboriginal Medical Service, Geraldton, Western Australia, Australia

\section{Twitter Tapan Rai @TapanRaiUTS and Erin Godecke @ErinGodecke}

Acknowledgements The authors would like to thank the Aboriginal participants in Missing Voices — the forerunner to this trial—whose recommendations were embedded in the current trial.
Contributors EA, NC, DH, JC, JMK, ST, LF, DAC, TR, EG, GJH, IL, CH, DW and ND are co-applicants on the funding application. All authors contributed to the development of the protocol. Principal Investigator EA wrote the first draft of the manuscript and all authors contributed to the editing of this manuscript. EA, LF, ST, JMK, EG and GJH provided design input; TR statistical analysis input; DAC economic analysis input; MM, EA, DH, NC, ND, JC, ST, IL, CH and DW intervention input; ST, JMK, DH and $\mathrm{EA}$ designed the process evaluation; $\mathrm{JC}, \mathrm{CH}$ and $\mathrm{DW}$ ensured cultural security in methodologies used; ND, EA, JMK, ST, NC and MM designed the translation plan.

Funding This work is supported by NHMRC Grant \#1132468 (2017-2022), Western Australian Department of Health, and the Royal Perth Hospital Medical Research Foundation, with the in-kind support of the Neurological Council of Western Australia, Bega Garnbirringu Health Services, Geraldton Regional Aboriginal Medical Service, Kimberley Aboriginal Medical Services Council and the Stroke Foundation.

Map disclaimer The depiction of boundaries on the map(s) in this article does not imply the expression of any opinion whatsoever on the part of BMJ (or any member of its group) concerning the legal status of any country, territory, jurisdiction or area or of its authorities. The map(s) are provided without any warranty of any kind, either express or implied.

\section{Competing interests None declared.}

Patient and public involvement Patients and/or the public were involved in the design, or conduct, or reporting, or dissemination plans of this research. Refer to the Methods section for further details.

Patient consent for publication Not required.

Provenance and peer review Not commissioned; externally peer reviewed.

Open access This is an open access article distributed in accordance with the Creative Commons Attribution Non Commercial (CC BY-NC 4.0) license, which permits others to distribute, remix, adapt, build upon this work non-commercially, and license their derivative works on different terms, provided the original work is properly cited, appropriate credit is given, any changes made indicated, and the use is non-commercial. See: http://creativecommons.org/licenses/by-nc/4.0/.

\section{ORCID iDs}

Elizabeth Armstrong http://orcid.org/0000-0003-4469-1117

Judith M Katzenellenbogen http://orcid.org/0000-0001-5287-5819

Dominique A Cadilhac http://orcid.org/0000-0001-8162-682X

Tapan Rai http://orcid.org/0000-0003-1610-8983

Erin Godecke http://orcid.org/0000-0002-7210-1295

\section{REFERENCES}

1 Australian Bureau of Statistics. Estimates of Aboriginal and Torres Strait Islander Australians. ABS cat. No. 3238.0.55.001, 2016. Available: https://www.abs.gov.au/AUSSTATS/abs@.nsf/DetailsPage/ 3238.0.55.001June\%202016?OpenDocument

2 Department of Communications,, Information Technology and the Arts. National Indigenous languages survey report 2005. ACT: Australian Institute of Aboriginal and Torres Strait Islander Studies, 2005.

3 Australian Government. Closing the gap report, 2020. Available: https://ctgreport.niaa.gov.au/sites/default/files/pdf/closing-the-gapreport-2020.pdf

4 Katzenellenbogen JM, Vos T, Somerford P, et al. Burden of stroke in Indigenous Western Australians: a study using data linkage. Stroke 2011;42:1515-21.

5 Katzenellenbogen JM, Atkins ER, Thompson SC, et al. Missing voices: profile and extent of acquired communication disorders in Aboriginal and non-Aboriginal adult stroke survivors in Western Australia using linked administrative records. Int J Stroke 2016;11:103-16.

6 Jamieson LM, Harrison JE, Berry JG. Hospitalisation for head injury due to assault among Indigenous and non-Indigenous Australians, July 1999--June 2005. Med J Aust 2008;188:576-9.

7 Katzenellenbogen JM, Atkins E, Thompson SC, et al. Missing voices: profile, extent, and 12-month outcomes of nonfatal traumatic brain injury in Aboriginal and non-Aboriginal adults in Western Australia using linked administrative records. J Head Trauma Rehabil 2018;33:412-23.

8 Esterman A, Thompson F, Fitts M, et al. Incidence of emergency department presentations for traumatic brain injury in Indigenous and non-Indigenous residents aged 15-64 over the 9-year period 20072015 in North Queensland, Australia. Inj Epidemiol 2018;5:40. 
9 Teoh V, Sims J, Milgrom J. Psychosocial predictors of quality of life in a sample of community-dwelling stroke survivors: a longitudinal study. Top Stroke Rehabil 2009;16:157-66.

10 Temkin NR, Corrigan JD, Dikmen SS, et al. Social functioning after traumatic brain injury. J Head Trauma Rehabil 2009;24:460-7.

11 Armstrong E, Coffin J, McAllister M, et al. 'I've got to row the boat on my own, more or less': aboriginal australian experiences of traumatic brain injury. Brain Impairment 2019;20:120-36.

12 Armstrong E, Coffin J, Hersh D, et al. "You felt like a prisoner in your own self, trapped": the experiences of Aboriginal people with acquired communication disorders. Disabil Rehabil 2021;43:1903-16.

13 Kelly J, Dwyer J, Pekarsky B. Managing two worlds together: Stage 2 - patient journey mapping tools. Melbourne: The Lowitja Institute, 2012.

14 Fitts MS, Bird K, Gilroy J, et al. A qualitative study on the transition support needs of Indigenous Australians following traumatic brain injury. Brain Impair 2019;20:137-59.

15 Kilkenny MF, Dewey HM, Sundararajan V, et al. Readmissions after stroke: linked data from the Australian stroke clinical Registry and hospital databases. Med J Aust 2015;203:102-6.

16 Zhao Y, Condon J, Lawton P, et al. Lifetime direct costs of stroke for Indigenous patients adjusted for comorbidities. Neurology 2016;87:458-65.

17 Kilkenny M, Lannin N, Kim J. Stroke care and outcomes for Australian Aboriginal and non-Aboriginal patients: observational study from the Australian stroke clinical Registry. Int J Stroke 2018;13:7.

18 Armstrong E, Hersh D, Hayward C, et al. Communication disorders after stroke in Aboriginal Australians. Disabil Rehabil 2015;37:1462-9.

19 Armstrong EM, Ciccone N, Hersh D, et al. Development of the Aboriginal communication assessment after brain injury (ACAABI): a screening tool for identifying acquired communication disorders in Aboriginal Australians. Int J Speech Lang Pathol 2017;19:297-308.

20 Armstrong E, McAllister M, Hersh D. A screening tool for acquired communication disorders in Aboriginal Australians after brain injury: lessons learned from the pilot phase. Aphasiology2019:1-25.

21 Coffin J. Rising to the challenge in Aboriginal health by creating cultural security. Aboriginal and Islander Health Worker Journal 2007;31:22-4.

22 Shahid S, Bessarab D, van Schaik KD, et al. Improving palliative care outcomes for Aboriginal Australians: service providers' perspectives. BMC Palliat Care 2013;12:26.

23 Hersh D, Armstrong E, Panak V, et al. Speech-language pathology practices with Indigenous Australians with acquired communication disorders. Int J Speech Lang Pathol 2015;17:74-85.

24 Barr VJ, Robinson S, Marin-Link B, et al. The expanded chronic care model: an integration of concepts and strategies from population health promotion and the chronic care model. Hosp Q 2003;7:73-82.

25 Laycock AF, Walker D, Harrison N. Researching Indigenous health: a practical guide for researchers. Carlton South, Victoria: The Lowitja Institute, 2011.

26 Gilroy J, Donelly M, Colmar S. Conceptual framework for policy and research development with Indigenous people with disabilities. Australian Aboriginal Studies 2013;2:42.

27 Nakata M. the Cultural Interface. The Australian Journal of Indigenous Education 2007:36:7-14.

28 EuroQol Group. EuroQol--a new facility for the measurement of health-related quality of life. Health Policy 1990;16:199-208.

29 van Swieten JC, Koudstaal PJ, Visser MC, et al. Interobserver agreement for the assessment of handicap in stroke patients. Stroke 1988;19:604-7.

30 Keith RA, Granger CV, Hamilton BB, et al. The functional independence measure: a new tool for rehabilitation. Adv Clin Rehabil 1987;1:6-18.

31 Thornton M, Travis SS. Analysis of the reliability of the modified caregiver strain index. J Gerontol B Psychol Sci Soc Sci 2003;58:S127-32.

32 Zigmond AS, Snaith RP. The hospital anxiety and depression scale. Acta Psychiatr Scand 1983;67:361-70.

33 Chan A-W, Tetzlaff JM, Gøtzsche PC, et al. Spirit 2013 explanation and elaboration: guidance for protocols of clinical trials. BMJ 2013;346:e7586.

34 Hemming K, Taljaard M, McKenzie JE, et al. Reporting of stepped wedge cluster randomised trials: extension of the CONSORT 2010 statement with explanation and elaboration. BMJ 2018;363:k1614.

35 Brown CA, Lilford RJ. The stepped wedge trial design: a systematic review. BMC Med Res Methodol 2006;6:54.

36 Skoss R, White J, Stanley M. Study protocol for a prospective process evaluation of a culturally secure rehabilitation program for
Aboriginal Australians after brain injury: the healing right way project. BMJ Open 2021;this issue.

37 Hoffmann TC, Glasziou PP, Boutron I, et al. Better reporting of interventions: template for intervention description and replication (TIDieR) checklist and guide. BMJ 2014;348:g1687.

38 Sacco RL, Kasner SE, Broderick JP, et al. An updated definition of stroke for the 21st century: a statement for healthcare professionals from the American heart Association/American stroke association. Stroke 2013;44:2064-89.

39 Koponen S, Taiminen T, Portin R, et al. Axis I and II psychiatric disorders after traumatic brain injury: a 30-year follow-up study. Am J Psychiatry 2002;159:1315-21.

40 National Institute of health stroke scale (NIHSS). Available: http:// www.stroke-site.org/stroke_scales/stroke_scales.html

41 Teasdale G, Jennett B. Assessment of coma and impaired consciousness. A practical scale. Lancet 1974;2:81-4.

42 Lin I, O'Sullivan P, Coffin J, et al. 'I can sit and talk to her': Aboriginal people, chronic low back pain and healthcare practitioner communication. Aust Fam Physician 2014;43:320-4.

43 Aboriginal Health Council of WA. The Aborginial community controlled health services model of care n.d. Available: https://kams. org.au/remote/acchs-model-of-care/

44 McCoy K, Chan H. A neurological integrated care pathway. Australasian Journal of Neuroscience 2015;25:25-9.

45 Vogler J, Klein A-M, Bender A. Long-Term health-related quality-oflife in patients with acquired brain injury and their caregivers. Brain Inj 2014;28:1381-8.

$46 \mathrm{Kim}$ S-K, Kim S-H, Jo M-W, et al. Estimation of minimally important differences in the EQ-5D and SF-6D indices and their utility in stroke. Health Qual Life Outcomes 2015;13:32.

47 Stroke Foundation. Clinical guidelines for stroke management Melbourne, Australia, 2017. Available: https://strokefoundation.org. au/What-we-do/Treatment-programs/Clinical-guidelines

48 Armstrong E, Hersh D, Katzenellebogen J. Enhancing personal factors. aphasia Rehabiliation best practice statements 2014: comprehensive supplement to the Australian aphasia rehabilitation pathway, 2014. Available: http://www.aphasiapathway.com.au/fluxcontent/aarp/pdf/2014-COMPREHENSIVE-FINAL-01-10-14-1.pdf

49 UK Medical Research Council. Developing and evaluating complex interventions. Medical Research Council, 2006. www.mrc.ac.uk/ complexinterventionsguidance

50 Faul F, Erdfelder E, Lang A-G, et al. G*Power 3: a flexible statistical power analysis program for the social, behavioral, and biomedical sciences. Behav Res Methods 2007;39:175-91.

51 Woertman W, de Hoop E, Moerbeek M, et al. Stepped wedge designs could reduce the required sample size in cluster randomized trials. J Clin Epidemiol 2013;66:752-8.

52 Husereau D, Drummond M, Petrou S, et al. Consolidated health economic evaluation reporting standards (cheers) statement. Int J Technol Assess Health Care 2013;29:117-22.

53 Cadilhac DA, Kim J, Wilson A, Berge E, et al. Improving economic evaluations in stroke: a report from the ESO health economics Working group. Eur Stroke J 2020;5:184-192.

54 Armstrong E, Hersh D, Katzenellenbogen JM, et al. Study Protocol: Missing Voices - Communication Difficulties after Stroke and Traumatic Brain Injury in Aboriginal Australians. Brain Impairment 2015;16:145-56.

55 The National Health and Medical Research Council,, the Australian Research Council and Universities Australia.. National statement on ethical conduct in human research 2007 (updated 2018. Canberra: Commonwealth of Australia, 2018.

56 Therapeutic Goods Administration. Note for guidance on good clinical practice (CPMP/ICH/135/95) and note for guidance on good clinical practice (CPMP/GCP/135/95) annotated with Therpeutic goods administration (TGA) comments (DSEB, July $2000,2000$.

57 National Health and Medical Research Council. Ethical conduct in research with Aboriginal and Torres Strait Islander peoples and communities: guidelines for researchers and stakeholders. Commonwealth of Australia: Canberra, 2018.

58 National Health and Medical Research Council. Keeping research on track II: a companion document to ethical conduct in research with Aboriginal and Torres Strait Islander peoples and communities: guidelines for researchers and stakeholders (2018). Canberra: Commonwealth of Australia, 2018.

59 Australian Institute of Aboriginal and Torres Strait Islander Studies (AIATSIS). AIATSIS code of ethics for Aboriginal and Torres Strait Islander research. Acton, ACT: Australian Institute of Aboriginal and Torres Strait Islander Studies, 2020. 